Www.jmscr.igmpublication.org Impact Factor 5.84

Index Copernicus Value: 83.27

ISSN (e)-2347-176x ISSN (p) 2455-0450

crossref DOI: https://dx.doi.org/10.18535/jmscr/v5i5.77

Journal Of Medical Science And Clinical Research

IGM Publication

An Official Publication of IGM Publication

\title{
Effect of pulsed mode Phonophoresis in relieving Inflammation and Pain in Supraspinatus tendinitis
}

\author{
Authors \\ Siva Kumar.B ${ }^{1}$, Dr Biju Ravindran ${ }^{2}$, R. Sreekar Kumar Reddy ${ }^{3}$, N.Vamsidhar \\ ${ }^{1}$ Assistant Professor, Narayana College of Physiotherapy, Nellore, A.P, India \\ ${ }^{2}$ Professor \& HOD, Narayana Medical College \& Hospital, Dept of Orthopaedics, Nellore, A.P, India \\ ${ }^{3}$ Professor, Narayana College of Physiotherapy, Nellore, A.P, India \\ ${ }^{4}$ Assistant Professor, Narayana College of Physiotherapy, Nellore, A.P, India
}

\begin{abstract}
Purpose: The aim of this study was to find the significance of pulsed mode ultrasound in reducing inflammation \& pain by using Ketoprofen as couplant.

Methods: 20 subjects presenting with supraspinatus tendinitis, aged 25-35years old, participated in this Study. Subjects were divided randomly into 2 groups.

Group $A=$ pulsed mode of ultrasound $(1.0 \mathrm{w} / \mathrm{cm} 2$ for 4 minutes $)$ with aqueous gel as couplant.

Group $B=$ pulsed mode of ultrasound $(1.0 \mathrm{w} / \mathrm{cm} 2$ for 4 minutes) with ketoprofen as couplant. 13

Visual Analogue Scale(VAS) and Range of Motion(ROM) is assessed pre treatment and immediately post treatment.

Result: The $p$ value of VAS (post treatment) and ROM (post treatment) in phonophoresis pulsed mode pvalue 0.05 than pulsed mode aqueous gel.

Conclusion: Pulsed mode of ultrasound along with ketoprofen as couplant shows better results compared to pulsed mode when aqueous gel is used as the couplant.

Keywords: Phonophoresis, Tendinitis, Ultrasound, Ketoprofen Gel.
\end{abstract}

\section{Introduction}

Supraspinatus tendinitis is a clinical syndrome in which there is pain in the shoulder during glenohumeral abduction.

It results in partial rupture of rotatory cuff \& calcific deposits, periarthritis, subacromion bursitis and also fracture associated with greater tubercle of humerus.

It also gives rise to impingement syndrome where impingement occurs below coracoacromial arch. Neer's stages of supraspinatous tendinitis:

1) stage of oedema
2) tendinitis \& fibrositis

3) rotatory cuff tears

4) bony changes.

Phonophoresis was first used to treat polyarthritis of the hand by delivery of hydrocortisone ointment into Inflamed areas in 1954. Since then it has been reported to be used in the treatment of various dermatological and musculoskeletal disorders. ${ }^{7}$

The mechanism by which ultrasound enhances the transdermal penetration of substances is not entirely clear. One could think of the vasodilation observed on macroscopic examination, but this 
would certainly not be enough on its own, since it does not imply any change of the waterproof keratin layer of the skin, which should necessarily be altered. ${ }^{8}$ Ketoprofen (proprionic acid derivative) in the form of $2.5 \%$ gel was chosen for the experiment for being a well-known and widely used nonsteroidal anti-inflammatory drug whose analgesic and anti inflammatory effects manifest quickly after administration. Evidence from clinical and pharmacological studies imply that Ketoprofen gel exerts its actions by inhibiting cycloxygenase (COX1\&2) enzymes thereby diminishing the production of proinflammatory prostaglandin precursors. ${ }^{13}$

\section{Reliability of the visual analogue scale for measurement of pain}

A VAS is measurement instrument that tries to measure a characteristic or attitude that is believed to range across a continuum of values and can't easily be directly measured. ${ }^{9}$ Operationally a VAS is usually a horizontal line, $100 \mathrm{~mm}$ in length, anchored by word descriptors at each end. The patient marks the line the point they feel which represents their perception of their current state. The VAS score is determined by measuring inn millimetres from left hand end of the line to the point that the patient marks. ${ }^{9}$

Reliability of the VAS for acute pain measurement as assessed by the ICC appears to be high. Ninety percent of the pain ratings were reproducible within ${ }^{9} \mathrm{~mm}$. These data suggest that the VAS is sufficiently reliable to be used to assess acute pain. $^{10}$

\section{Reliability of Goniometer}

The full-circle goniometer, or universal goniometer (UG), is a versatile device for recording measurements of peripheral joint ROM in healthy subjects and inpatients. Based on a clinical study of 60 patients with orthopaedic disorders in a physical therapy outpatient department, conclusion was drawn that AROM measurements on the cervical spine made by the same physical therapist have good to high reliability, regardless of whether the therapist used the CROM device or the UG. Repeated measurements with the UG had poor to fair between tester reliability. ${ }^{15}$

The purpose of this study is to compare the effectiveness of pulsed mode ultrasound in reducing inflammation \& pain by using Ketoprofen as couplant to pulsed mode ultrasound by using aqeous gel as couplant.

\section{Methodology \\ Number and Source}

20 subjects were taken from young \& middle aged population.

\section{Inclusion Criteria}

1) Male or Female with age of 25-35 years.

2) Subjects with supraspinatous tendinitis.

\section{Exclusion Criteria}

1. Subjects with musculoskeletal disorder other than supraspinatus tendinitis that would limit performance in these subjects.

2. Skin disorders which would irritate by eitherincrease in warmth of the part or by the lubricants which might be used, e.g. eczema.

3. In presence of malignant tumours.

4. In case of any previous fracture or surgery at neck.

5. All contraindications of ultrasonic therapy.

Method of selecting \& assigning subjects to groups 20 subjects having supraspinatus tendinitis were considered for this study. They were then screened to remove the subjects who did not fulfil the criteria for the study. After screening, the subjects they were randomly divided into two groups.

\section{Instruments and Tool used}

1. Ultrasound machine- Technomed Electroson-408

2. Ketoprofen gel

3. Aqueous gel

\section{Research Design}

It is an experimental design.

Variables

Independent variables- Ultrasonic Therapy 
Dependent variables- Visual Analogue Scale

\section{Procedure}

Subjects fulfilling the inclusion criteria were taken into consideration. The procedure was explained to the subjects and a written consent was taken after explaining the benefits and clearing the doubts of the subject regarding study. After pain level assessment by help of visual analogue scale (VAS) and Range of Motion using the universal goniometer they were randomly divided into two groups namely, A and B.

Group A = pulsed mode of ultrasound $(1.0 \mathrm{w} / \mathrm{cm} 2$ for 4 minutes) with aqeous gel as couplant.

Group B = pulsed mode of ultrasound $(1.0 \mathrm{w} / \mathrm{cm} 2$ for 4 minutes) with ketoprofen as couplant.

The ultrasound was given for 4 minutes at 1.0 w/cm2. After the treatment pain level and Range of Motion is taken again.

\section{Results}

A paired sample $\mathrm{t}$ test reveal a statistically reliabledifference between the mean number of VAS pre and post in phonophoresis mode $(\mathrm{M}=$ $6.7333, \mathrm{~s}=1.03280)$ and $(\mathrm{M}=3.9333, \mathrm{~s}=1.48645)$ that the $\mathrm{t}(14)=12.582, \mathrm{P}(\mathrm{a})=.000$ at two tail test. $\mathrm{A}$ paired sample $t$ test reveal a statistically reliable difference between the mean number of ROM pre and ROM post in phonophoresis mode $(\mathrm{M}=27.0000, \mathrm{~s}=5.29150)$ and $(\mathrm{M}=37.0667, \mathrm{~s}=$ 4.19977) that the $\mathrm{t}(14)=-11.093, \mathrm{P}(\mathrm{a})=.000$ at two tail test. A paired samples t test reveal a statistically reliable difference between the mean number of VAS pre and VAS post in pulsed mode $(\mathrm{M}=-6.8000, \mathrm{~s}=1.32017)$ and $(\mathrm{M}$ 4.4000, $\mathrm{s}=1.50238)$ that the, $\mathrm{t}(14)=8.806, \mathrm{P}(\mathrm{a})=.000$.at two tail test. A paired samples $t$ test reveal a statistically reliable difference between the mean number of ROM pre and ROM post in pulsed mode $(\mathrm{M}=-23.7333, \mathrm{~s}=10.73357) \quad$ and $\quad(\mathrm{M}=33.7333$, $\mathrm{s}=9.51290)$ that the, $\mathrm{t}(14)=-10.569, \mathrm{P}(\mathrm{a})=.000$. at two tail test.

An independent-samples t-test was conducted to compare VAS post treatment in pulsed mode with phonophoresis and pulsed mode. There was a significant difference in the scores for pulsed
$(\mathrm{M}=4.4, \quad \mathrm{SD}=1.5) \quad$ and $\quad(\mathrm{M}=3.93, \quad \mathrm{SD}=1.48)$ conditions; $\mathrm{t}(28)=0.855, \mathrm{p}=0.400$. The result suggest that VAS decreases more with phonophoresis mode than in pulsed mode.

An independent-samples t-test was conducted to compare ROM post treatment in phonophoresis mode and pulsed mode.

There was a significant difference in the scores for pulsed $(\mathrm{M}=33.73, \mathrm{SD}=9.51)$ and phonophoresis $(\mathrm{M}=37.06, \quad \mathrm{SD}=4.19) \quad$ conditions; $\mathrm{t}(28)=-1.24$, $\mathrm{p}=0.225$.

The result suggest that ROM increases more in pulsed mode ultrasound with Ketoprofen as couplant.

Hence it can be said that pulsed mode with Ketoprofen couplant has more effect rather than using aqueous gel in pulsed mode. Mild heating has the effect of reducing pain and promoting healing process. Kramer(1987), investigating the increase in conduction velocity in motor and sensory nerves following therapeutic

ultrasound, concluded that this was likely to be related to the heating effect of ultrasound. ${ }^{12}$

Ketoprofen in the form of $2.5 \%$ gel was chosen for the experiment for being a well-known and widely used nonsteroidal anti-inflammatory drug whose analgesic and anti inflammatory effects manifest quickly after administration. Evidence from clinical and pharmacological studies imply that Ketoprofen gel exerts its actions by inhibiting cycloxygenase (COX1\&2) enzymes thereby diminishing the production of proinflammatory prostaglandin precursors.

The mechanism by which ultrasound enhances the transdermal penetration of substances is not entirely clear. One could think of the vasodilation observed on macroscopic examination, but this would certainly not be enough on its own, since it does not imply any change of the waterproof keratin layer of the skin, which should necessarily be altered. $^{18}$ No volunteers presented any complication of any kind nor did they report any discomfort with the treatment at any time, all of them resuming normal life immediately after the end of treatment Apart from slight redness and a temperature increase on touch, no sign of local 
irritation was detected by macroscopic inspection of the irradiated areas.

\section{Discussion}

According to the unpaired $t$ test done between post values of VAS in case of phonophoresismode and pulsed mode the $p$ value is $<0.005$. The post value of ROM in phonophoresis mode and pulsed mode the $\mathrm{p}$ value is $<0.005$.

In this study, the clinical efficacy of ketoprofen gel as a coupling agent using pulsed mode of was compared with pulsed mode.

According to the study, by using phonophoresis mode of ultrasound there is more pain relive rather than by using pulsed mode.

Phonophoresis mode has been recommended for muscular cellular disorders such as tendinitis, joint stiffness or pain whereas pulsed mode is preferred for soft tissue repair. ${ }^{12}$

This could be because in pulsed mode the time average intensity is reduced which reduces the amount of energy available to heat the tissues while ensuring that the energy available in each pulse is high enough for mechanical or non-thermal effects rather than the thermal effects to predominate. $^{12}$

Mild heating has the effect of reducing pain and tendinitis and promoting healing process. Kramer (1987), investigating the increase in conduction velocity in motor and sensory nerves following therapeutic ultrasound, concluded that this was likely to be related to the heating effect of ultrasound. $^{12}$

ketoprofen is a very commonly used nonsteroidal anti-inflammatory drug (NSAIDs). It is an analgesic-anti-inflammatory drug, similar in efficacy to naproxen. It inhibits PG synthesis and is somewhat COX-2 selective. ${ }^{13}$

The mechanism by which ultrasound enhances the transdermal penetration of substances is not entirely clear. One could think of the vasodilation observed on macroscopic examination, but this would certainly not be enough on its own, since it does not imply any change of the waterproof keratin layer of the skin, which should necessarily be altered. ${ }^{18}$ No volunteers presented any complication of any kind nor did they report any discomfort with the treatment at any time, all of them resuming normal life immediately after the end of treatment Apart from slight redness and a temperature increase on touch, no sign of local irritation was detected by macroscopic inspection of the irradiated areas. ${ }^{8}$

\section{Conclusion}

Pulsed mode of ultrasound along with ketoprofen as couplant shows better results in reducing inflammation \& pain by inhibiting COX-1 \& COX-2 enzymes thereby diminishing the production of proinflammatory prostaglandin precursors compared to pulsed mode when aqueous gel is used as the couplant.

\section{References}

1. Ali Gur; Physical Therapy Modalities in Management of Fibromyalgia; Current Pharmaceutical Design, 2006 12, 29-35

2. Haraldsson B, Gross A, Myers CD, Ezzo J, Morien A, Goldsmith $\mathrm{CH}$, Peloso PMJ, Brønfort G, Cervical Overview Group. Massage for mechanical neck disorders. Cochrane Database of Systematic Reviews 2006, Issue 3. Art. No.: CD004871. DOI: 10.1002/14651858.CD004871.pub3

3. Sheila Kitchen, Electrotherapy Evidence-based Practice, Eleventh Edition, pg 221-228

4. Sangita Chakrabarty, Md, Msph, Roger Zoorob, Md, Mph; Fibromyalgia; American Family Physician; Volume 76, Number 2 July 15, 2007

5. Sunday Akinbo, Oluwatoyosi Owoeye, Sunday Adesegun; Comparison of the Therapeutic Efficacy of ketoprofen Phonophoresis in the Management of Knee Osteoarthritis; Turk J Rheumatol 2011;26(2):111-119

6. Peter Croft, Jonathan Burt, Joanna Schollum, Elaine Thomas, Gary Macfarlane, Alan Silman; More pain, more tender points: is fibromyalgia just one end 
of a continuous spectrum?; Annals of the Rheumatic Diseases 1996; 55: 482-485

7. Giovana c. Rosim, Cláudio Henrique Barbieri, Fernando Mauro Lanças, and Nilton Mazzer; Phonophoresis In Human Volunteers; Ultrasound in Med. \& Biol., Vol. 31 No. 3 pp. 337- 343, 2005

8. Russell Rothenberg, MD; Fibromayalgia, pathophysiology and treatment; Fibromyalgia frontiers, 2010, vol. 18, No. 1

9. John low BA(Hons.), FCSP, DipTP, SRP, Ann Reed BA, MCSP, DipTP, SRP; Electrptherapt Explained Principles and Practice, 3rd edition, pg 172-196

10. KD Tripathi; Essentials of Medical Pharmacology; 6th edition; page 184-194

11. Bijur PE, Silver W, Gallagher EJ.; Reliability of the visual analog scale for measurement of acute pain; Acad Emerg Med. 2001 Dec;8(12):1153-7

12. Kamal Dua, V.K.Sharma, UVS Sara, D.K.Agrawal, M.V.Ramana; Penetration Enhancers for TDDS: A Tale of the Under Skin Travelers; Adv. in Nat. Appl. Sci., 3(1): 95-101, 2009

13. Mazières, B; Rouanet, S; Guillon, Y; Scarsi, C; Reiner, V (2005). "Topical ketoprofen patch in the treatment of tendinitis: a randomized, double blind, placebo controlled study.". The Journal of rheumatology 32 (8): 1563-70. 